\title{
Postprandial lipid absorption in seven heterozygous carriers of deleterious variants of MTTP in two abetalipoproteinemic families
}

\author{
Mathilde Di Filippo, PharmD, PhD*, Mathilde Varret, PhD, Vanessa Boehm, MD, \\ Jean-Pierre Rabès, MD, PhD, Latifa Ferkdadji, MD, Laurent Abramowitz, MD, \\ Sabrina Dumont, Catherine Lenaerts, MD, Catherine Boileau, PharmD, PhD, \\ Francisca Joly, MD, PhD, Jacques Schmitz, MD, PhD, \\ Marie-Elisabeth Samson-Bouma, PhD, Dominique Bonnefont-Rousselot, PharmD, PhD
}

UF Dyslipidemies, Service de Biochimie et Biologie moléculaire Grand Est, GHE, Hospices Civils de Lyon, Bron Cedex, France (Drs Di Filippo and Dumont); Univ-Lyon, CarMeN laboratory, Inserm U1060, INRA U1397, Université Claude Bernard Lyon 1, INSA Lyon, Villeurbanne, France (Dr Di Filippo); INSERM U1148, Université Paris Diderot, Hôpital Bichat-Claude Bernard, Paris Cedex 18, France (Drs Varret, Rabès, Boileau, and Samson Bouma); Service de gastroentérologie, MICI et Assistance Nutritive, Hopital Beaujon, Hopital Beaujon (AP-HP), Université Paris VII, Paris, France. INSERM UMR1149, Centre de Recherche sur l'Inflammation Paris Montmartre (CRI), Paris, France (Drs Boehm and Joly); AP-HP, HUPIFO, Hôpital Ambroise Paré, Laboratoire de Biochimie et Génétique Moléculaire \& UVSQ, UFR des Sciences de la Santé Simone Veil, Montigny-Le-Bretonneux, France (Dr Rabès); Service d'anatomie et de cytologie pathologiques, Hôpital Robert Debré, Université Paris 7, Paris, France (Dr Ferkdadji); Service d'HépatoGastroentérologie, Hôpital Bichat-Claude Bernard, Assistance Publique-Hôpitaux de Paris (AP-HP), Paris Cedex 18, France (Dr Abramowitz); Service de Pédiatrie, CHU d'Amiens, Amiens (Dr Lenaerts); Département de Gastroentérologie pédiatrique, Hopital Necker-Enfants Malades, Paris, France (Dr Schmitz); Service de Biochimie métabolique, Hôpitaux universitaires Pitié-Salpêtrière-Charles Foix (AP-HP), Paris, France (Dr Bonnefont-Rousselot); and Faculté de Pharmacie de Paris, Université Paris Descartes, Sorbonne Paris Cité, Unité de Technologies Chimiques et Biologiques pour la Santé, U 1022 INSERM, UMR 8258 CNRS, Paris, France (Dr Bonnefont-Rousselot)

\section{KEYWORDS:}

Genetic

hypocholesterolemia;

Abetalipoproteinemia;

Microsomal triglyceride

transfer protein;

Fat malabsorption;

Postprandial;
BACKGROUND: Abetalipoproteinemia, a recessive disease resulting from deleterious variants in MTTP (microsomal triglyceride transfer protein), is characterized by undetectable concentrations of apolipoprotein B, extremely low levels of low-density lipoprotein cholesterol in the plasma, and a total inability to export apolipoprotein B-containing lipoproteins from both the intestine and the liver.

OBJECTIVE: To study lipid absorption after a fat load and liver function in 7 heterozygous relatives from 2 abetalipoproteinemic families, 1 previously unreported.

RESULTS: Both patients are compound heterozygotes for p.(Arg540His) and either c.708_709del p.(His236Glnfs*11) or c.1344+3_1344+6del on the MTTP gene. The previously undescribed patient

\footnotetext{
* Corresponding author. Mathilde Di Filippo, UF Dyslipidémies, Laboratoire de Biologie Médicale Multi Sites, Centre de Biologie et de Pathologie Est, Service de biochimie et biologie moléculaire, 59 Bd Pinel, F-69677 Bron, France.
}

E-mail address: mathilde.di-filippo@chu-lyon.fr

Submitted June 25, 2018. Accepted for publication October 12, 2018. 
Liver steatosis; Apolipoprotein B has been followed for 22 years with ultrastructure analyses of both the intestine and the liver. In these 2 families, 5 relatives were heterozygous for p.(Arg540His), 1 for p.(His236Glnfs*11) and 1 for c.1344+3_1344+6del. In 4 heterozygous relatives, the lipid absorption was normal independent of the MTTP variant. In contrast, in 3 of them, the increase in triglyceride levels after fat load was abnormal. These subjects were additionally heterozygous carriers of Asp2213 APOB in-frame deletion, near the cytidine mRNA editing site, which is essential for intestinal apoB48 production. Liver function appeared to be normal in all the heterozygotes except for one who exhibited liver steatosis for unexplained reasons.

CONCLUSION: Our study suggests that a single copy of the MTTP gene may be sufficient for human normal lipid absorption, except when associated with an additional APOB gene alteration. The hepatic steatosis reported in 1 patient emphasizes the need for liver function tests in all heterozygotes until the level of risk is established.

\section{Introduction}

Abetalipoproteinemia (ABL; OMIM 200100) is one of the 2 very rare inherited diseases characterized by very low or undetectable apolipoprotein (apo) B-containing lipoprotein $(\mathrm{LpB})$ levels in blood. ABL has an autosomal recessive mode of inheritance, which distinguishes it from familial homozygous hypobetalipoproteinemia (OMIM 107730 commonly named Ho-FHBL) segregating as an autosomal dominant trait. ABL, as Ho-FHBL, is associated with a lipid malabsorption syndrome and the inability to secrete $\mathrm{LpB}$. The similar phenotypes in both disorders arise, in $\mathrm{ABL}$, from deleterious variants in the large polypeptide subunit of microsomal triglyceride (TG) transfer protein (MTP) and, in Ho-FHBL, from deleterious variants in the $A P O B$ gene.

MTP has been shown to be essential for LpB assembly and secretion as well as for further lipid absorption processing. MTP-mediated lipid transfer is the limiting step in the assembly and secretion of LpB: in vitro studies have demonstrated that apoB secretion is proportional to MTP activity. ${ }^{1}$ In mice, partial MTP deficiency reduces TG absorption and apoB100 levels in plasma, and it increases the accumulation of cytosolic fat in the liver. ${ }^{2,3}$ In ABL patients, the absence of MTP activity disrupts TG-rich LpB assembly in both the intestine and the liver, which leads to a complete inability to secrete postprandial chylomicrons (CMs) and very low density lipoproteins, respectively. Endoscopy shows a white coating of the small intestine. The enterocytes are overloaded with large lipid droplets in the cytoplasm, which appear, at the ultrastructural level, to be non-membrane-bound. Affected individuals, thus, develop essentially a lipid malabsorption syndrome with fat-soluble vitamin deficiency. In most of these patients, liver function is altered and steatosis may be associated with elevated transaminases. In some cases, evolution to fibrosis and cirrhosis has been reported. ${ }^{4-6}$ Patients with ABL exhibit profound hypocholesterolemia, hypotriglyceridemia, the absence of $\mathrm{LpB}$, and no CMs and apoB48 after a fat load. ${ }^{6}$

Heterozygous carriers of MTTP deleterious variants have not been believed to exhibit clinical or biological abnormalities, in general, or abnormalities in the lipid profile, in particular. ${ }^{7}$ Indeed, almost all of the obligate heterozygotes have been reported to have normal lipid levels. ${ }^{6,8,9}$ However, to our knowledge, there have been no studies of fat loading. ${ }^{8}$ The effects of deleterious variants in 1 allele of MTTP on intestinal absorption and the liver are unknown. Furthermore, common variants in the MTTP gene have been reported to predispose to non-alcoholic steatohepatitis in the general population including in heterozygous subjects. ${ }^{10,11}$ These observations raise the question of a potential risk of fat malabsorption and hepatic complications in heterozygous carriers of deleterious MTTP variants.

In this article, we evaluated the impact, in 7 heterozygous relatives from $2 \mathrm{ABL}$ families, of MTTP single deleterious allele variants on hepatic function and lipid absorption after a fat load.

\section{Material and methods}

\section{Subjects}

Members from 2 unrelated French families were evaluated (Fig. 1). The proband from family A (A.III.2) has not been reported, whereas the 1 from family B (II.1) has been partially described previously., ${ }^{6,12}$

Blood samples and intestinal and hepatic biopsies were collected from family A's proband and normal control subjects (for intestinal biopsies) using the procedures and the experimental methods approved by INSERM (RBM 0256) and by a bioethics committee (Comité Consultatif de Protection des Personnes dans la Recherche Biomédicale de Paris Bichat-Claude Bernard, Paris, France, CCPPRB Bichat-C. Bernard-2003/05). Informed written consent was obtained from all participants or from their legal guardians.

\section{Genetic analysis}

After the extraction of genomic DNA from the blood, encoding regions and the flanking intronic junctions of the MTTP, APOB, PCSK9, LDLR (only exon 7), ANGPTL3, $S A R I B, F A B P 1$, and SARIA genes were amplified by 


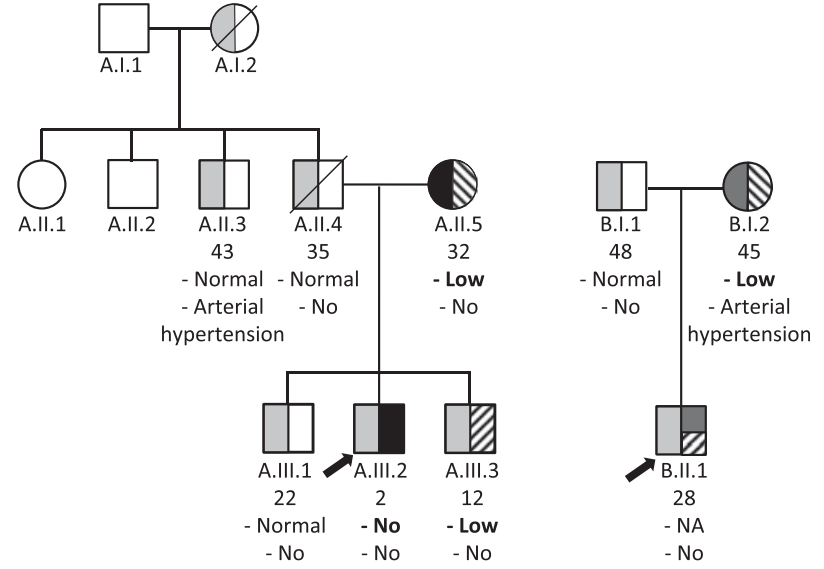

Figure 1 Pedigrees of the families. Symbols divided in halves indicate heterozygous carriers of variants: light gray symbols indicate the MTTP c.1619G $>$ A p.(Arg540His), black symbols the MTTP c.708_709del or p.(His236Glnfs*11), dark gray symbols the $M T T P$ c.1344+3_1344+6del, and hatched symbols represent the $A P O B$ c.6639_6641del. For each subject, after the unique identification number and the age (years) are indicated, 1 below the other, lipid absorption and comorbidities at the time of fat load. NA, not available.

polymerase chain reaction. The amplicons were directly sequenced on an ABI PRISM DNA sequencer (Applied Biosystems) as previously described. ${ }^{6,13,14}$

\section{Analysis of intestinal and hepatic biopsies}

Histological and ultrastructural analyses of both intestinal and liver biopsies from the patient from family A were performed after a 12- to 15-hour fast and treated as described previously. ${ }^{4}$

\section{Analysis of lipids and lipoproteins after a fast or an oral fat load}

Venous blood samples were drawn after a 12-hour fast and after a lipid load. The lipid load for the young proband of family A (A.III.2) was performed as previously described. ${ }^{15}$ For all the relatives, a $4520-\mathrm{kJ}$ meal was given at T0 immediately after drawing the fasting blood samples. The meal contained $74 \%$ lipids with $72 \%$ saturated, $25 \%$ monounsaturated, and $3 \%$ polyunsaturated fatty acids. Typically, it was composed of $200 \mathrm{~mL}$ of unskimmed milk with sweetener, $80 \mathrm{~g}$ of bread with $60 \mathrm{~g}$ of butter, and $100 \mathrm{~g}$ of cream with sweetener. The effect of the fat load on plasma TG concentration, which reflects lipid absorption ability, is expressed as the percentage of maximal increase in TG concentration, during at least 5 hours, except for III.2 (90 minute) and II.3 (190 minute). We evaluated quantitatively the absorption by calculating the total area under the curve from TG concentrations, using the trapezoidal rule. ${ }^{16}$ However, the area under the curves (corrected or not for the basal TG value) was difficult to interpret. Indeed, oral fat loads were performed at different periods in the heterozygous subjects, with different times of follow-up, even if the fat load was always the same. To surmount these difficulties, we decided to use the maximal percentage increase in TG concentration taking into account the absolute TG concentrations (basal and during the fat load). In the same way, this process of calculation decreased the variability due to the different methods of TG determination throughout the 22-year follow-up.

\section{Results}

\section{Abetalipoproteinemic patients}

The pedigrees of the 2 families are shown in Figure 1. The clinical and biological description of the unreported proband and updated information for the previously described case $^{6,12}$ are provided in Tables 1 and 2, respectively.

\section{Family A}

The proband (A.III.2) is a boy born on November 01, 1993 after an uneventful pregnancy. He was the second of 3 boys of unrelated healthy Caucasian parents. From normal French curves, the birth weight and height of the patient were, respectively, $4100 \mathrm{~g}(+1 \mathrm{SD})$, and $52 \mathrm{~cm}$ (+1 SD). He was breastfed during the first 6 weeks of life. The child presented with regurgitations and vomiting followed by loose stools in the first week of life. At 6 months of age, the height was below the normal French curves $(63 \mathrm{~cm},-1.5 \mathrm{SD})$ and weight average for height $(6.5 \mathrm{~kg})$. He was followed during 22 years. All along these years, he exhibited the usual previously described clinical and biochemical features of ABL except for an unusual hypertonicity at 7 months (Table 1). Serum TG, total cholesterol, and low-density lipoprotein cholesterol (LDL-C) levels were very low as were the fat-soluble vitamins. These vitamin deficiencies persisted throughout the 22-year follow-up. There was poor compliance to a low-fat diet and to vitamin E supplementation (Table 2). Acanthocytosis was present. Cardiac and ophthalmic investigations were normal as was psychomotor development.

Gastrointestinal endoscopies were performed in 1995, 1996, and 2006, all after a 12-hour fast. A typical white stippling-like hoar frosting covering the mucosal surface of the duodenojejunal part of the intestine was observed on each occasion. Histologically, whenever obtained, all the biopsies exhibited a mucosa with a normal thickness and villi of normal height. The enterocytes were always distended with a cytoplasm clarified due to the presence of numerous vacuoles that predominated in the upper twothirds of the villi, often on 1 side, as previously described (data not shown). ${ }^{15}$ Electron microscopy always showed non-membrane-bound lipid droplets in the cytoplasm 


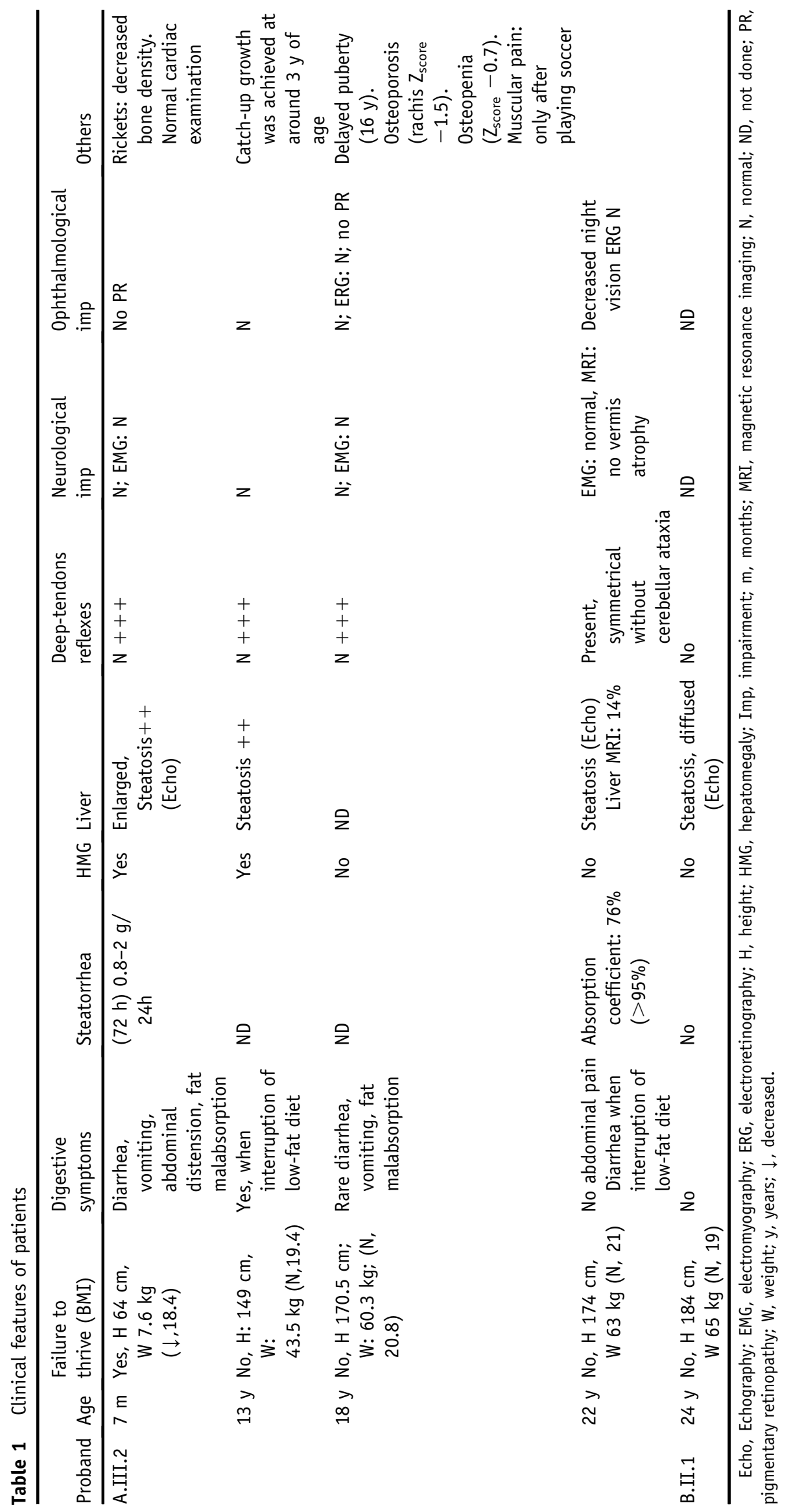




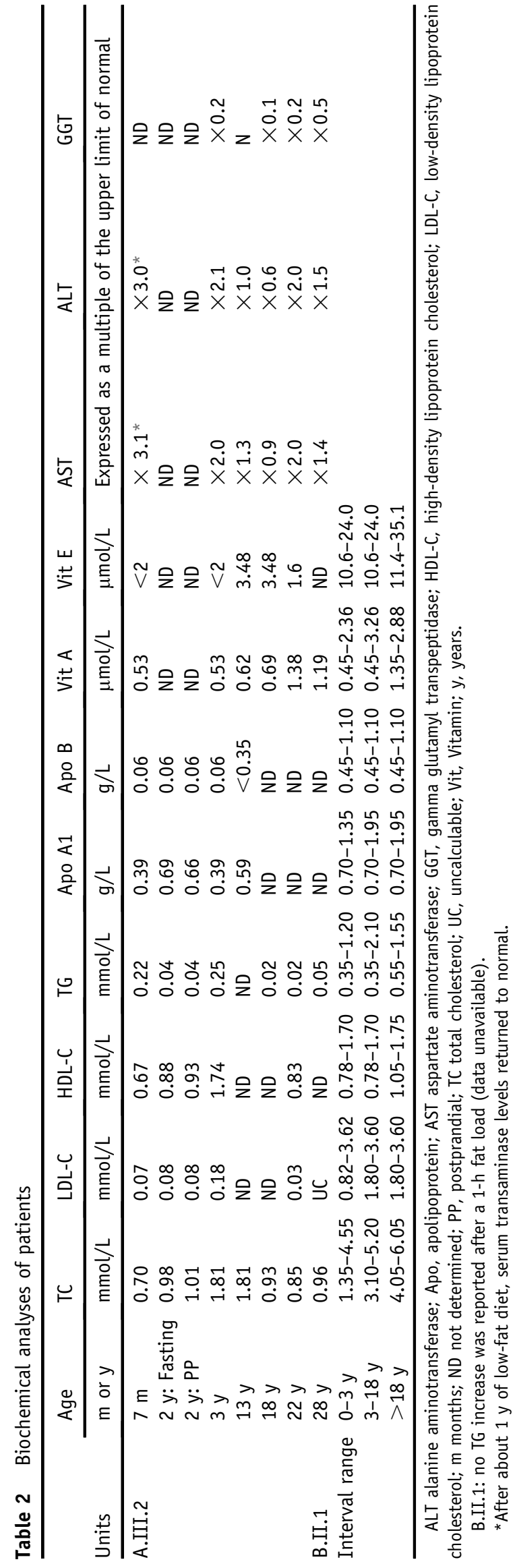

(Fig. 2A and B). No CM-like structures were observed in the basolateral spaces (Fig. 2A). The Golgi cisternae in the enterocytes from the proband were dilated but empty (Fig. 2B) in contrast with the flat Golgi apparatus in enterocytes from normal fasted individuals (Fig. 2C).

Liver biopsies were performed in 1996 and 2006 to follow up the steatosis severity. In 1996, there was hepatic macrovacuolar steatosis up to $90 \%$ of the parenchymal tissue, associated with portal and centrolobular fibrosis. No cirrhosis was observed (data not shown). Electron microscopy showed hepatocytes with marked accumulation of lipid droplets, which were not membrane bound (Fig. 2D). In 2006, the same massive centrolobular macrovacuolar steatosis and microvacuolar steatosis were present although in a decreased amount (15\%) and fibrosis had disappeared (Fig. 2E).

Based on the clinical aspects, the laboratory data, the typical aspect of the intestinal and hepatic biopsies as well as the normolipidemic parents, the patient was suspected to have ABL at about 1 year of age and further confirmed by MTTP gene sequencing (see below). A low-fat diet and Intralipid infusions were thus prescribed from then up to age 13 years and orally thereafter. During this period, an oral complementation of liposoluble vitamins was prescribed (TOCO 500, 2-4 g/d). Lipid infusions were reintroduced every month in March 2016 with $1 \mathrm{~g}$ of vitamin E, $50,000 \mathrm{IU}$ of vitamin A, $10 \mathrm{mg}$ of vitamin $\mathrm{K}$ in $250 \mathrm{~mL}$ of $20 \%$ Smoflipid (containing soybean oil, medium-chain TG, olive oil, and fish oil).

At age 24 years, despite a poor compliance to vitamin $\mathrm{E}$ supplementation and permanently low vitamin E levels $(<3.5 \mu \mathrm{mol} / \mathrm{L})$, neurological examination was normal: symmetrical and unusually brisk tendon reflexes without ataxia (Tables 1 and 2). Indeed, throughout the 22-year follow-up, the only striking clinical feature was the constant hypertonicity. To our knowledge, this is the first report of such a remarkable absence of neurological complications in a typical ABL patient.

\section{Family B}

The clinical features of the proband of family B (subject B.II.1, boy born on November 22, 1987) were previously described by Wetterau et al. and Rheberg et al. and have been summarized and updated (to 26 years of age) by Di Filippo et al. ${ }^{6,12}$ Infusion of Intralipid every 3 weeks (with $45 \mathrm{mg}$ of vitamin $\mathrm{K}, 500 \mathrm{mg}$ of vitamin $\mathrm{E}$, and 50,000 UI of vitamin A) has been prescribed between 6 months of age and until 2011. In 2011, infusions of Intralipid (soya oil) were replaced by ClinOleic infusions (olive oil: $160 \mathrm{mg} / \mathrm{mL}$, soy oil: $40 \mathrm{mg} / \mathrm{mL}$ ). Despite a low-fat diet and liposoluble vitamin substitution, deep tendon reflexes were absent. At 28 years of age, the biological data were essentially unchanged except for a slight increase of liver cytolysis (1.4 and 1.5 times the upper limit of normal for aspartate aminotransferase and alanine aminotransferase, respectively) with diffuse moderate steatosis (Tables 1 and 2). 

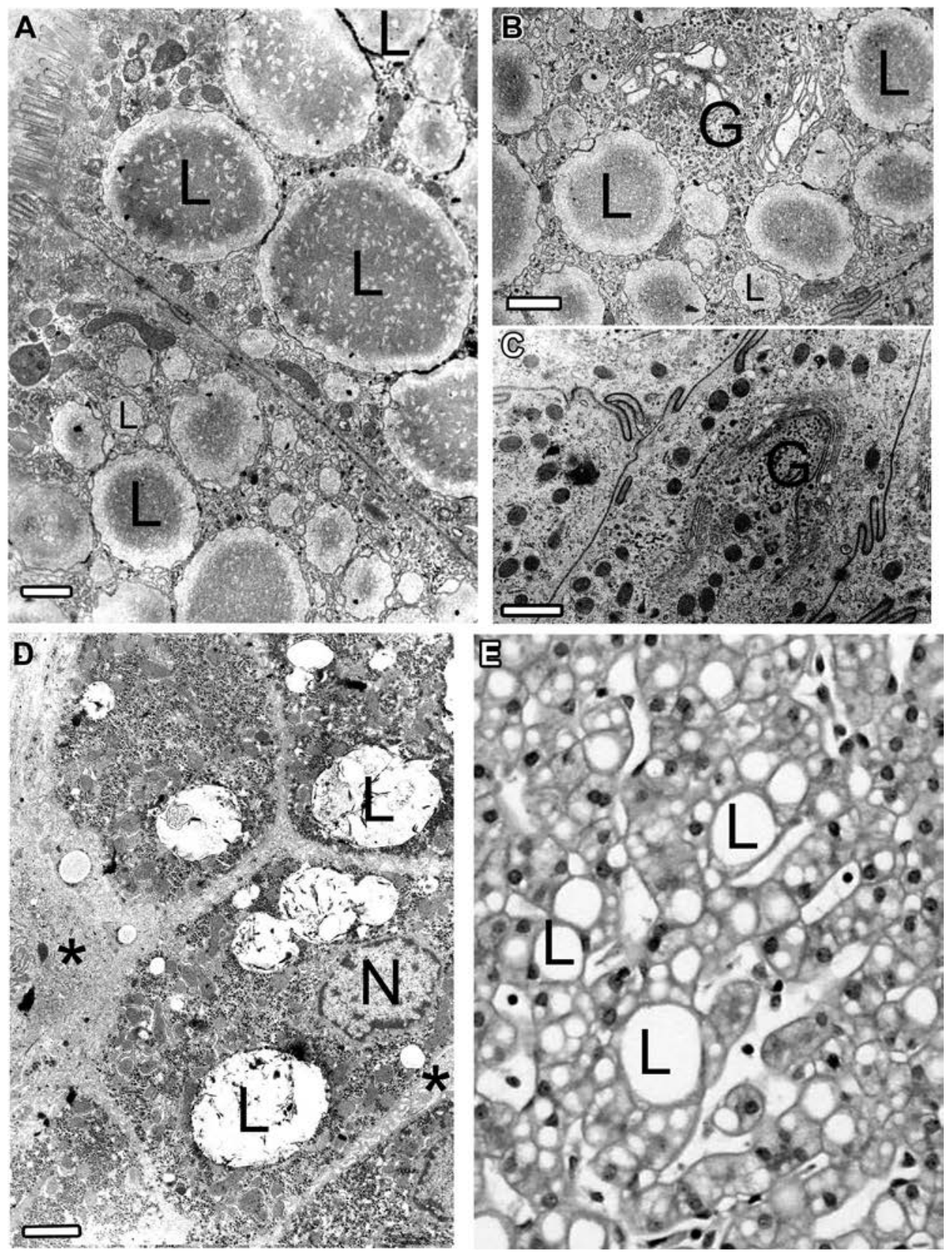

Figure 2 Typical aspect of intestinal biopsies from family A's proband (subject A.III.2) (A and B) and from a normal subject (C). Typical aspect of hepatic biopsies from family A's proband (D and E). Electron microscopy of the intestinal biopsy exhibits the accumulation within the cytoplasm of numerous lipid droplets, free in the cytoplasm and which have diameters from 0.3 to $7 \mu \mathrm{m}$ (Fig. $2 \mathrm{~A}$ and B, labeled L, 1995). The Golgi apparatus (Fig. 2B, labeled G, 1995) is distended but without lipoprotein-like material inside. This is in contrast to the flattened Golgi in fasted enterocytes of a normal subject (Fig. 2C, labeled G). Ultrastructural (Fig. 2D and E, 1996) and histological (Fig. 2E, 2006) analysis of the liver showed that it was always engorged with numerous free cytoplasmic lipid droplets (Fig. 2D and E, labeled L), with diameters up to $8 \mu \mathrm{m}$. In 1996, intercellular spaces were enlarged, with fibrous tracts extending into the hepatic parenchyma, reflecting the fibrosis (Fig. 2D, dark arrows). In 2006, fibrosis disappeared (Fig. 2E, labeled L). The bars represent $1 \mu \mathrm{m}$.

\section{Genetic diagnosis of abetalipoproteinemia}

\section{Family A}

Family A's proband (subject A.III.2, Fig. 1) is an unreported case of ABL who exhibits compound heterozygous deleterious variants in the MTTP gene. The first variant is a previously described substitution in exon 13, NM_000253.3(MTTP):c.1619G $>$ A or p.(Arg540His). The frequency of this allele (rs199422220) is $0.0014 \%$ in the gno$\mathrm{mAD}$ database (frequencies in Americans: $0.0014 \%$ and in non-Finnish Europeans: 0.0024\%). This variant was inherited from the patient's father (Fig. 1). The proband's 2 healthy 
brothers, one of his uncles and his grandmother (Fig. 1), are heterozygous for this variant. ${ }^{6,12,17}$ The second deleterious variant is a 2 bp deletion in exon 7, NM_000253.3 (MTTP):c.708_709del or p.(His236Glnfs*11), which leads to a frame shift and a premature stop codon. This variant is not described in the gnomAD database (allele frequency below 1/270,000) and was reported once in ABL in the ClinVar database. This variant is inherited from his mother and is absent in the rest of the family (Fig. 1).

Because loss of function variants in PCSK9 have been reported to be associated with hypocholesterolemia, ${ }^{18}$ we sequenced the gene in this patient. No causative variants were observed in this gene nor in exon 7 of the $L D L R$ gene, which encodes the PCSK9-interacting domain. ${ }^{19}$

\section{Family B}

Family B's proband (subject B.II.1, Fig. 1) was previously shown, using cDNA from intestinal biopsies, to be a compound heterozygote for an in-frame deletion of exon 11 (new nomenclature) and p.(Arg540His). ${ }^{12}$ Direct sequencing of the genomic DNA from this family was, thus, directed to exons 11 and 13. The p.(Arg540His) mutation is inherited from his father (Fig. 1). The second mutation, a new variant, which is not described in the gnomAD database or in ClinVar, was inherited from his mother, and is a 4 bp deletion: NM_000253.3(MTTP):c.1344+3_1344+6del downstream from the donor site (Fig. 1). An effect on splicing is predicted, in silico, for this variant with a decreased score for the exon 11's donor site ranging from $-24 \%$ for Human Splicing Finder to a complete abolition for MaxEntScan and NNSplice software.

\section{Fasting and postprandial lipid analyses}

\section{Probands}

In the fasting state, at diagnosis and during the followup, both probands exhibited a marked fasting hypocholesterolemia, with a dramatic decrease in LDL-C and apoB concentrations but a high-density lipoprotein cholesterol level within the normal range (Table 2). Serum lipid electrophoresis showed the absence of CMs, undetectable beta and prebeta lipoproteins and normal alpha lipoproteins (data not shown).

In the postprandial state $(30$ and 90 minute after fat load), in both probands, serum TG did not increase and CMs were undetectable. Serum lipid electrophoresis was unaltered as compared to the fasting state (data not shown).

\section{Fasting and postprandial lipid analyses of the hetero- zygous relatives in families $A$ and $B$}

Fasting lipid values were interpreted using reference values for the laboratory (see Tables).

Fasting TG values of the heterozygous members of both families were in the normal range for 5 subjects (A.II.4, A.III.1, A.III.3, B.I.1, and B.I.2), increased in 1 subject (A.II.3) and decreased in subject A.II.5. Total cholesterol was within the normal range for all subjects (see Table 3).

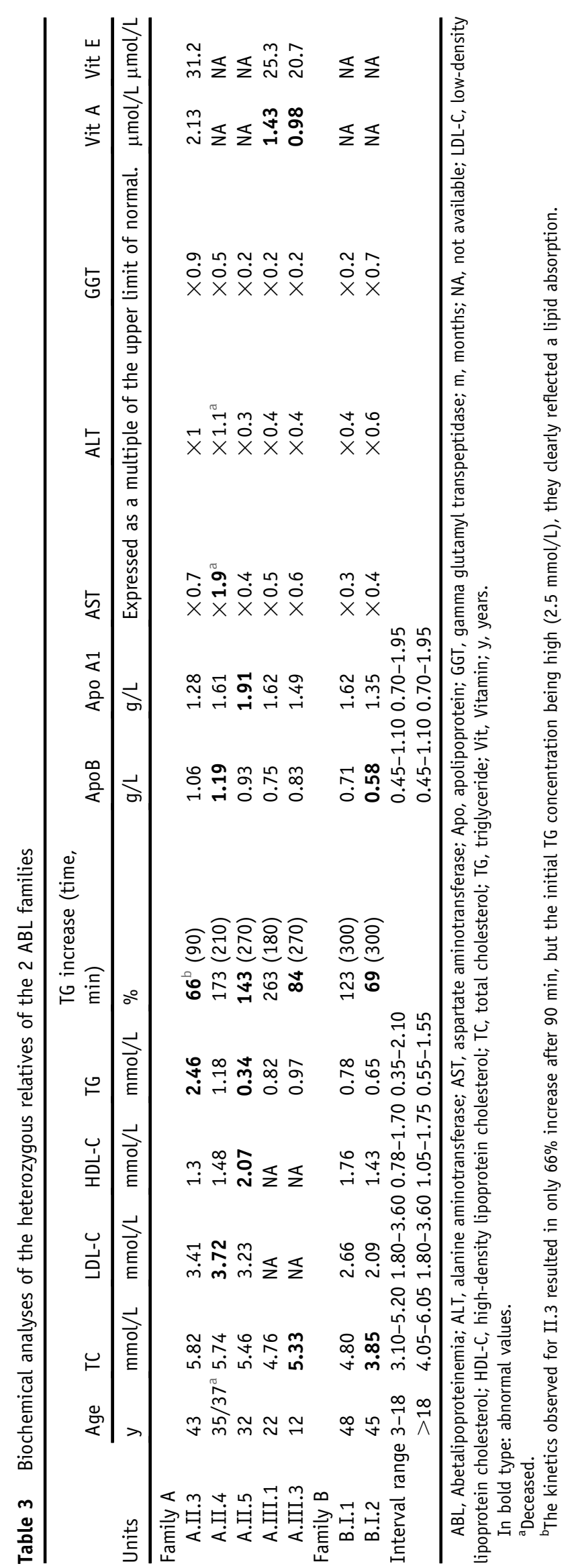


After a fat load, analysis of the TG values was based on the kinetics reported for normal individuals by Nakajima et al. ${ }^{20}$ Two groups of relatives were identified (Fig. 3): in 1 group, the father (subject A.II.4), brother (subject A.III.1), and uncle (subject A.II.3) in family A and TG levels clearly rose after intake of a fat meal, and CMs clearly appeared upon serum electrophoresis. The percentages of maximal increase in TG concentrations appeared after 210 and 180 minutes for A.II.4 and A.III.1, respectively, with respective values of $173 \%$ and $263 \%$. This increase was of the same order of magnitude as that observed by Nakajima et al. ${ }^{20}$ for control subjects, which reflects normal lipid absorption. For the father of family B (subject B.I.1), the $123 \%$ increase in TG concentration after 300 minutes closer to normal values was considered as normal. The kinetics observed for A.II.3 resulted in only a $66 \%$ increase after 90 minutes, but the initial TG concentration was high $(2.5 \mathrm{mmol} / \mathrm{L})$. The results clearly indicate lipid absorption.

In contrast, another group, the mother and the brother in family A (subjects A.II.5 and A.III.3), as well as the mother in family B (subject B.I.2), exhibited an opalescent serum with slightly increased TG levels. However, CMs were not detectable upon serum electrophoresis. Furthermore, it is important to note that abdominal pain associated with diarrhea was reported quickly after the fat load, which is consistent with lipid malabsorption syndrome. The maximal increases in TG concentrations were clearly lower for B.I.2 and A.III.3 than those observed by Nakajima et al. ${ }^{20}$ because they were only 69 and $84 \%$, after 300 and 270 minute, respectively. For A.II.5, the apparent normal increase in value (143\% after 270 minute) needs to be interpreted in light of the constant very low TG concentrations, always lower than $0.85 \mathrm{mmol} / \mathrm{L}$.

\section{Exploration of genes involved in chylomicron metabolism}

In an attempt to understand the origin of the decreased fat loading response of 3 of the 7 heterozygous carriers of deleterious variants in the $M T T P$ gene, we sequenced the $A P O B, A N G P T L 3, M T T P, S A R 1 B, F A B P 1$, and SARIA genes (known to be involved in LpB processing) in these individuals. It is important to note that each of these 7 heterozygotes shared different MTTP variants.

Subjects A.II.5 and A.III.3 in family A and subjects B.I.2 and B.II.1 from family B were heterozygous for the NM_000384.2(APOB):c.6639_6641del, an in-frame deletion in the $A P O B$ gene, which leads to the absence of residue 2213, the p.(Asp2213del). This in-frame variant has been described previously as rs541497967 in gnomAD with a minor allele frequency of $0.52 \%$ and of $0.61 \%$ in the non-Finnish European population. This deletion was found to be deleterious in silico (Provean score -9.8) (Provean http://sift.jcvi.org/).

Subjects B.I.2 and B.II.1 from family B were found to be homozygous and heterozygous carriers, respectively, for

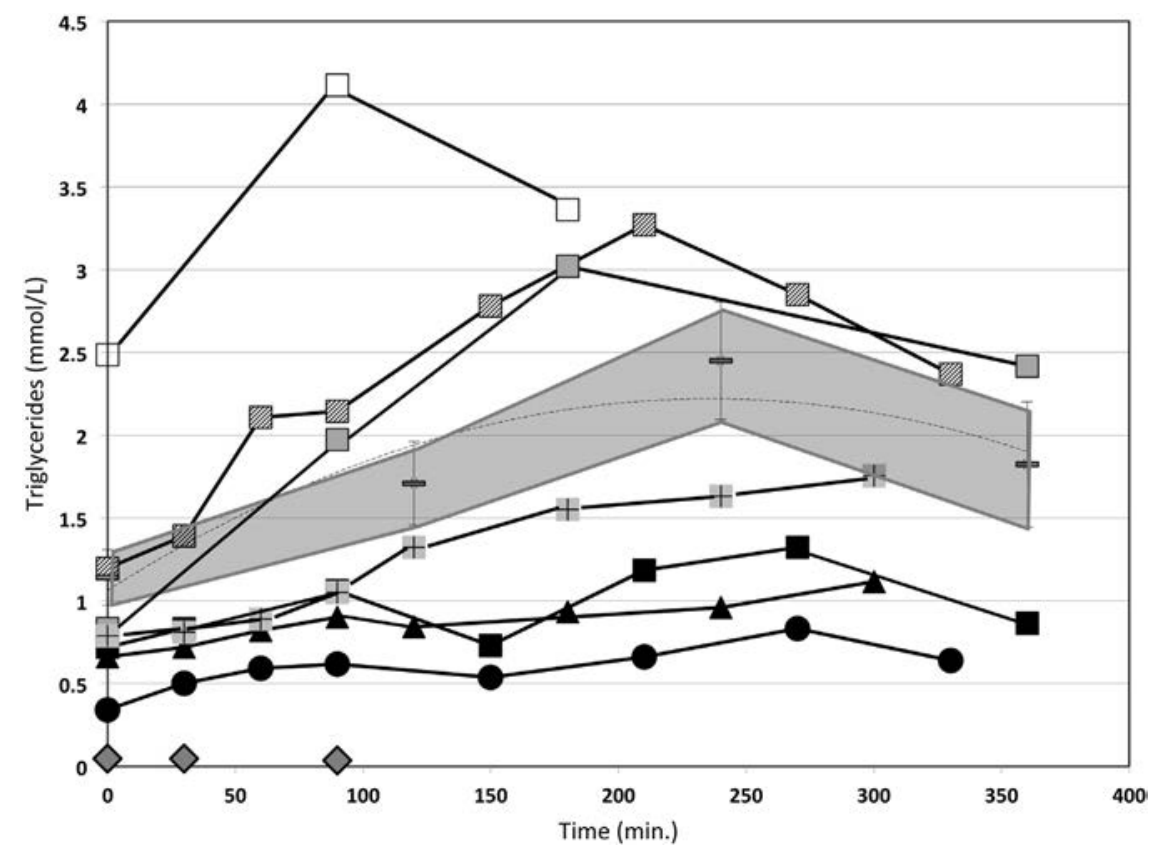

Figure 3 Changes in the serum concentration of triglycerides (TG) after the oral fat load. The TG kinetics from the proband (A.III.2, gray diamond), father (A.II.4, diagonal hatched square), mother (A.II.5, black round), uncle, (A.II.3, white square), and brothers (A.III.1, gray square and A.III.3, black square) of family A's proband, and the mother (B.I.2, black triangle) and father (B.I.1, gray square with black cross) of the family B's proband are shown. Squares represent heterozygous carriers of the c.1619G $>$ A p.(Arg540His) variant, the round symbol is a carrier of the c.708_709del or p.(His236Glnfs*11) variant, and the triangle is the carrier of the c.1344+3_1344+6del variant on $M T T P$. Black symbols represent carriers of the $A P O B$ c.6639_6641del. The TG kinetics reported by Nakajima et al. ${ }^{20}$ after an oral fat load in normal individuals appear in gray area (mean \pm SEM). 
the NM_001443.2:c.280A $>$ G, a common missense variant, p.(Thr94Ala) in the FABPl gene. This variant was previously described as rs2241883 in gnomAD with an minor allele frequency of $30.5 \%$. This variant affects a moderately conserved residue and the Grantham distance is low. It is considered as tolerated in silico using SIFT (score 0.15) and PolyPhen-2 (score 0).

In the 3 heterozygous carriers of deleterious MTTP variants (subjects A.II.5 and A.III.3 from family A, and subject B.I.2 from family B), no other point deleterious variant was found in these other genes.

\section{Liver function in the heterozygous relatives of the 2 families}

In family A, as shown in Table 3, the mother (subject A.II.5), the 2 brothers, (subjects A.III.1 and A.III.3), and the uncle (subject B.II.3) exhibited normal serum liver function tests. Furthermore, on ultrasound examination, the mother (subject A.II.5), the uncle (subject A.II.3), and a brother (subject A.III.3) showed normal liver echogenicity.

In contrast, the father (subject A.II.4), considered as healthy, neither diabetic nor obese nor an alcohol consumer, died suddenly from cardiac arrest in 2000. His liver was taken for transplantation. Pretransplant ultrasound of the liver showed a homogeneous hyperechogenic hypertrophic right lobe. Serum liver function tests were normal for alanine aminotransferase, GGT, alkaline phosphatase. Aminotransferase activity was slightly elevated (68 vs 35 IU/L for the normal value). A liver biopsy obtained during the transplantation exhibited a mixed microvesicular and macrovesicular steatosis in $70 \%$ of the hepatocytes in the centrolobular and periportal areas, without fibrosis. In addition, scattered polynuclear neutrophils were observed throughout the hepatic sinusoid. This liver steatosis was not correlated with major increased transaminase levels but was suggested by its hyperechogenicity and was confirmed by the biopsy reflecting precisely the liver fat load as in other contexts. $^{21}$

In family B, the father (subject B.I.1) and the mother (subject B.I.2) had normal serum liver function tests. Upon ultrasound examination, both showed normal liver echogenicity.

\section{Discussion}

We report in this article lipid absorption and liver function analyses in 7 heterozygous relatives from 2 different ABL families, 1 previously described for which we completed the molecular diagnosis ${ }^{6,12}$ and another 1 previously undescribed. This patient described here has been followed for 22 years. The most striking clinical event is the constant hypertonicity, despite poor compliance to vitamin $\mathrm{E}$ supplementation.

Because, to our knowledge, no information is available in the literature concerning lipid absorption in heterozygous carriers of deleterious $M T T P$ variants, we investigated this point in these 2 families, which include 5 carriers of the same MTTP variant. Indeed, in contrast to Hz-FHBL, it is believed that obligate heterozygotes for ABL, as expected from an autosomal recessive transmission, exhibit no clinical or laboratory abnormalities and, particularly, no plasma lipid abnormalities. ${ }^{7} \mathrm{We}$, thus, evaluated the impact of the MTTP mutation on 1 allele by studying fat loading and hepatic function in the 7 heterozygous relatives of these ABL patients.

Genetic analysis of the MTTP gene in these families showed that $\mathrm{ABL}$ is caused by 3 different deleterious variants. The first missense variant is the previously described p.(Arg540His) variant ${ }^{6,12,17}$, which leads, in vitro, to an absence of dimerization with protein disulphide isomerase and to the absence of MTP activity and, in vivo, to an absence of LpB secretion. ${ }^{12,22}$ Moreover, a significant reduction of MTP activity was observed for other missense variants occurring on the same residue, which lead to the loss of positive charge and change in hydrophobicity. ${ }^{23}$ This information supports the hypothesis that this residue, which is supposed to form a salt bridge with protein disulphide isomerase, constitutes a critical domain for lipid-transfer activity. ${ }^{22-24}$ This finding of 2 apparently unrelated families carrying the same missense variant, in addition to a family from Ontario of Northern European ancestry ${ }^{25}$ raises the question of a founder variation, as shown for p.(G865X) in the Ashkenazi Jewish population. ${ }^{26}$ The second variant is a $2 \mathrm{bp}$ deletion leading to a frame shift and a premature termination of the protein (p.His236Glnfs*11). Therefore, this variant is likely to be functionally defective. The third variant is a 4 bp deletion: c. $1344+3 \_1344+6$ del downstream from the donor site. In silico algorithms predicted an effect on splicing. The perfect in-frame deletion of exon 11 described by Rehberg et $\mathrm{al}^{12}$ on cDNA extracted from intestinal biopsies of this patient confirmed the effect of this variant on splicing.

In heterozygous relatives in these families, as previously reported in the literature, fasted lipid levels were found to be normal except for a slight decrease of TG in 1 subject (family A, subject A.II.5) and an increase in subject A.II.3 from family A. ${ }^{6,7,14,27-35}$ However, in some articles, about $17 \%$ and $14 \%$ (see supplemental material) of heterozygotes have been reported to have, respectively, elevated ${ }^{27,36}$ or low plasma triglycerides, total or LDL-C levels, ${ }^{28,31-33}$ but there is no information about the coexistence of other genetic or environmental factors, which could influence lipid levels.

Because, to our knowledge, no evaluation of fat loading in heterozygous carriers of deleterious MTTP variants have been reported to date, oral fat loads were performed in all the $7 \mathrm{ABL}$ heterozygotes. We show here that 4 heterozygous carriers of the MTTP p.(Arg540His) mutation in family A (subjects A.II.3 and A.II.4, and subject A.III.1, Fig. 1), and subject B.I.1 in family B, exhibit a normal response to a fat load.

Taken together, these data suggest that there is an adaptation in the heterozygous state with an increase in 
the synthesis of MTP by the normal allele or that MTP activity is present in excess in the normal state, with a single allele being able to provide a sufficient level of activity. ${ }^{7,8,37}$ In contrast, 3 other heterozygous carriers of deleterious MTTP variants (subjects A.II.5 and A.III.3 in family A and subject B.I.2 in family B) exhibited decreased postprandial TG responses. To explain these different responses, we additionally sequenced 6 other major genes known to be involved in the different steps of LpB assembly and secretion. We thus identified an additional in-frame deletion, the c.6639_6641del p.(Asp2213del) variant, in $A P O B$. This variant, previously found in patients exhibiting hypocholesterolemia, mixed hyperlipidemia, hypercholesterolemia as well as in control subjects, was consequently considered as nondeleterious by Alves et al. ${ }^{38,39}$ However, these nucleotides 6639 to 6641 are located near the « 6666 » cytidine mRNA editing site known to be crucial for the production of the normally occurring apoB48 in the intestine (nucleotide number 6538 in the new nomenclature). ${ }^{40}$ Thus, perhaps this variant might, therefore, disturb mRNA editing, blocking intestinal LpB secretion. Unfortunately, the apoB48 levels could not be measured after the fat load in this study. Further studies, such as evaluation of the response to a fat load in other carriers of only this $A P O B$ variant as well as in vitro experiments, such as pulse chase studies of apoB secretion in cells stably expressing wild-type or mutant forms of human apoB48, would be useful to evaluate the precise role of this $A P O B$ variant in human apoB48 secretion.

A liver biopsy, the gold standard for the evaluation of liver function and a biopsy was obtained in the context of transplanted liver for one of the 7 heterozygous adult carriers of 1 deleterious MTTP variant, the proband's father (subject A.II.4). To date, the causal link between this MTTP variant and liver steatosis has not been firmly established despite literature data reporting that common SNPs in the MTTP gene predispose to non-alcoholic steatohepatitis in the general population by impairing TG-rich lipoprotein export due to a lower transcriptional activity of MTP. ${ }^{10,11,41}$ Similarly, a cross-sectional study reported an association of the p.(Gln297His) in MTTP with a reduced serum cholesterol but an increased risk of nonalcoholic fatty liver disease. ${ }^{42}$ The observation of a clinically and biologically silent steatosis in subject A.II.2 suggests that liver steatosis and fibrosis should be monitored in heterozygous carriers of deleterious $M T T P$ variants. Liver biopsy being proscribed for ethical reasons, the noninvasive approach (FibroTest and FibroScan), could be recommended.

In the present study, we, thus, have investigated 7 heterozygous carriers of MTTP deleterious gene variants. In the literature, a great deal of information about the functions of MTP and its role in LpB assembly and secretion has derived from the study of mouse models. However, only a few of them have investigated the consequences of a partial decrease in activity of MTP.,43-45 Furthermore, extrapolation to humans has been found to be difficult because MTP expression and, consequently, hepatic TG secretion depends on the animal model. ${ }^{46}$ Due to the key role of MTP in LpB secretion, MTP inhibitors have been developed to block the assembly of metabolic precursors of LDL particles. Thus, studies of Lomitapide have shown that efficient titration of this inhibitor decreases LDL-C $50 \%$ to $90 \%{ }^{47,48}$ However, side effects have been noted such as diarrhea, decreased vitamin $\mathrm{E}$ and fatty acid levels, and increased fat liver content as assessed by nuclear magnetic resonance spectroscopy and liver biopsy. ${ }^{47-49}$ A case of progression of steatohepatitis to fibrosis also has been reported in a patient with pancreatitis who exhibited fatty liver before treatment. ${ }^{50}$ Unfortunately, due to ethical considerations, MTP mRNA, protein or activity levels in liver and intestine have not been measured. Thus, these data are not available in our study.

The limited number of patients and relatives is an evident limitation for our conclusion. This is due to the extreme rarity of the disorder. The incidence of the ABL disease is reported as less than 1 in $1,000,000 .^{51}$ To our knowledge, fewer than 65 families have been described worldwide. ${ }^{6}$ However, the study of these 2 families has provided additional knowledge, which contributes to a better understanding of these rare diseases. Indeed, we report here 1 previously undescribed patient who exhibited a negative fat load test, as described in 4 typical ABL cases with known MTTP mutations ${ }^{6,36,52}$ and the first description of fat load tests in heterozygous carriers of MTTP deleterious variants.

Despite this limitation, we report first that $4 / 7$ heterozygous carriers of deleterious MTTP variants have normal lipid absorption without known additional risks. Second, and perhaps more important, some heterozygotes, despite normal fasting lipid levels and without clinical abnormalities, do not absorb normally. In these heterozygous carriers of MTTP variants, other deleterious variants of genes involved in the LpB secretory mechanism, such as apoB reported here, or environmental factors should be evaluated. Furthermore, 1 subject was found with a clinically and biologically silent steatosis. The results of this study suggest that in ABL families, there should be (1) an obligatory cascade screening to identify all heterozygous carriers, (2) an evaluation of fasting lipid status with additional fat load lipid absorption studies of heterozygotes, and (3) liver monitoring using noninvasive tests in all heterozygotes. Additional subjects need to be investigated to confirm our results, ascertain the level of risk, and definitively establish appropriate treatment and screening.

\section{Acknowledgments}

The authors would like to express first, special thanks to Dr Lawrence Aggerbeck for his helpful discussion and reviewing of the article (Present address: INSERM UMR 1124, Toxicologie, pharmacologie et signalisation cellulaire Center Universitaire des Saints-Pères, 75006 Paris, France). 
The authors would like to thank Pr E Bruckert and his physicians and nurses for postprandial studies (Endocrinology Department) and Dr Yann Nadjar (Unité Neurogénétique et métabolisme, Département de Neurologie) for neurological investigation, both from Pitié-Salpêtrière hospital, AP-HP, 75013 Paris, Pr F Gottrand (Service de pédiatrie - Unité de Gastroentérologie, CHRU de Lille - Hôpital Jeanne de Flandre, 59037 Lille Cedex France), Pr Nicole Brousse (Service d'Anatomie et de Cytologie Pathologiques, Hôpital Necker-Enfants Malades, AP-HP, 75015 Paris, France), and Dr E Leteurtre (Service d'Anatomie et de Cytologie Pathologiques, Center Hospitalier et Universitaire de 59045 Lille, France) for medical pediatric follow-up.

The authors would like to thank Dr Isabel Beucler and Marie-Christine Federspiel for lipid laboratory investigation and technical assistance, respectively (Laboratoire des lipides, Service de Biochimie, Pitié-Salpêtrière hospital, AP-HP, 75013 Paris, France), Dr Nathalie Varroqueaux and Nicole Verthier (INSERM U 327, 75018 Paris, France) for her contribution and participation in the patient's studies and the histological and ultrastructural studies, respectively.

Authors' contributions: L.A., V.B., F.J., C.L., and J.S. contributed to clinical assessments; C.B., D.B-R., M.D.F., L.F., J-P.R., M-E.S-B., J.S., and M.V. conceived and designed the experiments. S.D. performed the experiments. C.B., D.B-R., M.D.F., J-P.R., M-E.S-B., J.S., and M.V. analyzed the data and provided critical reading of the article. V.B., D.B-R., M.D.F., M-E.S-B., and M.V. wrote the article.

\section{Financial disclosure}

The authors have no conflicts of interest to disclose.

\section{References}

1. Jamil H, Chu CH, Dickson JK Jr., et al Evidence that microsomal triglyceride transfer protein is limiting in the production of apolipoprotein B-containing lipoproteins in hepatic cells. J Lipid Res. 1998;39: $1448-1454$.

2. Raabe M, Flynn LM, Zlot CH, et al. Knockout of the abetalipoproteinemia gene in mice: reduced lipoprotein secretion in heterozygotes and embryonic lethality in homozygotes. Proc Natl Acad Sci U S A. 1998;95:8686-8691.

3. Iqbal J, Li X, Chang BH, et al. An intrinsic gut leptin-melanocortin pathway modulates intestinal microsomal triglyceride transfer protein and lipid absorption. J Lipid Res. 2010;51:1929-1942.

4. Bouma ME, Beucler I, Pessah M, et al. Description of two different patients with abetalipoproteinemia: synthesis of a normal-sized apolipoprotein B-48 in intestinal organ culture. J Lipid Res. 1990; 31:1-15.

5. Braegger CP, Belli DC, Mentha G, Steinmann B. Persistence of the intestinal defect in abetalipoproteinaemia after liver transplantation. Eur J Pediatr. 1998;157:576-578.
6. Di Filippo M, Moulin P, Roy P, et al. Homozygous MTTP and APOB mutations may lead to hepatic steatosis and fibrosis despite metabolic differences in congenital hypocholesterolemia. J Hepatol. 2014;61: 891-902.

7. Berriot-Varoqueaux N, Aggerbeck LP, Samson-Bouma M, Wetterau JR. The role of the microsomal triglygeride transfer protein in abetalipoproteinemia. Апnи Rev Nutr. 2000;20:663-697.

8. Lee J, Hegele RA. Abetalipoproteinemia and homozygous hypobetalipoproteinemia: a framework for diagnosis and management. J Inherit Metab Dis. 2014;37:333-339.

9. Welty FK. Hypobetalipoproteinemia and abetalipoproteinemia. Curr Opin Lipidol. 2014;25:161-168.

10. Rubin D, Schneider-Muntau A, Klapper M, et al. Functional analysis of promoter variants in the microsomal triglyceride transfer protein (MTTP) gene. Hum Mutat. 2008;29:123-129.

11. Peng XE, Wu YL, Lu QQ, Hu ZJ, Lin X. MTTP polymorphisms and susceptibility to non-alcoholic fatty liver disease in a Han Chinese population. Liver Int. 2014;34:118-128.

12. Rehberg EF, Samson-Bouma ME, Kienzle B, et al. A novel abetalipoproteinemia genotype. Identification of a missense mutation in the 97$\mathrm{kDa}$ subunit of the microsomal triglyceride transfer protein that prevents complex formation with protein disulfide isomerase. J Biol Chem. 1996; 271:29945-29952.

13. Charcosset M, Sassolas A, Peretti N, et al. Anderson or chylomicron retention disease: molecular impact of five mutations in the SAR1B gene on the structure and the functionality of Sar $1 \mathrm{~b}$ protein. Mol Genet Metab. 2008;93:74-84.

14. Di Filippo M, Crehalet H, Samson-Bouma ME, et al. Molecular and functional analysis of two new MTTP gene mutations in an atypical case of abetalipoproteinemia. J Lipid Res. 2012;53:548-555.

15. Georges A, Bonneau J, Bonnefont-Rousselot D, et al. Molecular analysis and intestinal expression of SAR1 genes and proteins in Anderson's disease (Chylomicron retention disease). Orphanet J Rare Dis. 2011;6:1.

16. Matthews JN, Altman DG, Campbell MJ, Royston P. Analysis of serial measurements in medical research. BMJ. 1990;300:230-235.

17. Wetterau JR, Aggerbeck LP, Bouma ME, et al. Absence of microsomal triglyceride transfer protein in individuals with abetalipoproteinemia. Science. 1992;258:999-1001.

18. Cohen J, Pertsemlidis A, Kotowski IK, Graham R, Garcia CK, Hobbs HH. Low LDL cholesterol in individuals of African descent resulting from frequent nonsense mutations in PCSK9. Nat Genet. 2005; 37:161-165.

19. Zhang DW, Lagace TA, Garuti R, et al. Binding of proprotein convertase subtilisin/kexin type 9 to epidermal growth factor-like repeat A of low density lipoprotein receptor decreases receptor recycling and increases degradation. J Biol Chem. 2007;282:18602-18612.

20. Nakajima K, Nakano T, Tokita Y, et al. Postprandial lipoprotein metabolism: VLDL vs chylomicrons. Clin Chim Acta. 2011;412: 1306-1318.

21. Burgert TS, Taksali SE, Dziura J, et al. Alanine aminotransferase levels and fatty liver in childhood obesity: associations with insulin resistance, adiponectin, and visceral fat. J Clin Endocrinol Metab. 2006;91:4287-4294.

22. Khatun I, Walsh MT, Hussain MM. Loss of both phospholipid and triglyceride transfer activities of microsomal triglyceride transfer protein in abetalipoproteinemia. J Lipid Res. 2013;54:1541-1549.

23. Miller SA, Burnett JR, Leonis MA, McKnight CJ, van Bockxmeer FM, Hooper AJ. Novel missense MTTP gene mutations causing abetalipoproteinemia. Biochim Biophys Acta. 2014;1842: $1548-1554$.

24. Mann CJ, Anderson TA, Read J, et al. The structure of vitellogenin provides a molecular model for the assembly and secretion of atherogenic lipoproteins. J Mol Biol. 1999;285:391-408.

25. Wang Y, Tran K, Yao Z. The activity of microsomal triglyceride transfer protein is essential for accumulation of triglyceride within microsomes in McA-RH7777 cells. A unified model for the assembly of very low density lipoproteins. J Biol Chem. 1999;274:27793-27800. 
26. Benayoun L, Granot E, Rizel L, Allon-Shalev S, Behar DM, Ben-Yosef T. Abetalipoproteinemia in Israel: evidence for a founder mutation in the Ashkenazi Jewish population and a contiguous gene deletion in an Arab patient. Mol Genet Metab. 2007;90:453-457.

27. Di Leo E, Lancellotti S, Penacchioni JY, et al. Mutations in MTP gene in abeta- and hypobeta-lipoproteinemia. Atherosclerosis. 2005;180:311-318.

28. Najah M, Di Leo E, Awatef J, et al. Identification of patients with abetalipoproteinemia and homozygous familial hypobetalipoproteinemia in Tunisia. Clin Chim Acta. 2009;401:51-56.

29. Uslu N, Gurakan F, Yuce A, Demir H, Tarugi P. Abetalipoproteinemia in an infant with severe clinical phenotype and a novel mutation. Turk J Pediatr. 2010;52:73-77.

30. Pons V, Rolland C, Nauze M, et al. A severe form of abetalipoproteinemia caused by new splicing mutations of microsomal triglyceride transfer protein (MTTP). Hum Mutat. 2011;32:751-759.

31. Sani MN, Sabbaghian M, Mahjoob F, Cefalu AB, Averna MR, Rezaei N. Identification of a novel mutation of MTP gene in a patient with abetalipoproteinemia. Ann Hepatol. 2011;10:221-226.

32. Mohamed N, Mohamed Youssef S, Mohamed Yahia H, et al. Molecular characterization of Tunisian families with abetalipoproteinemia and identification of a novel mutation in MTTP gene. Diagn Pathol. 2013;8:54.

33. Magnolo L, Najah M, Fancello T, et al. Novel mutations in SAR1B and MTTP genes in Tunisian children with chylomicron retention disease and abetalipoproteinemia. Gene. 2013;512:28-34.

34. Walsh MT, Iqbal J, Josekutty J, et al. Novel abetalipoproteinemia missense mutation highlights the importance of the N-terminal betabarrel in microsomal triglyceride transfer protein function. Circ Cardiovasc Genet. 2015;8:677-687.

35. Gunduz M, Ozaydin E, Atar MB, et al. Microsomal triglyceride transfer protein gene mutations in Turkish children: a novel mutation and clinical follow up. Indian J Gastroenterol. 2016;35:236-241.

36. Narcisi TM, Shoulders CC, Chester SA, et al. Mutations of the microsomal triglyceride-transfer-protein gene in abetalipoproteinemia. Am J Hum Genet. 1995;57:1298-1310.

37. Hooper AJ, Burnett JR, Watts GF. Contemporary aspects of the biology and therapeutic regulation of the microsomal triglyceride transfer protein. Circ Res. 2015;116:193-205.

38. Alves AC, Etxebarria A, Soutar AK, Martin C, Bourbon M. Novel functional APOB mutations outside LDL-binding region causing familial hypercholesterolaemia. Hum Mol Genet. 2014;23:1817-1828.

39. Johansen CT, Dube JB, Loyzer MN, et al. LipidSeq: a next-generation clinical resequencing panel for monogenic dyslipidemias. J Lipid Res. 2014;55:765-772.
40. Blanc V, Davidson NO. APOBEC-1-mediated RNA editing. Wiley Interdiscip Rev Syst Biol Med. 2010;2:594-602.

41. Bernard S, Touzet S, Personne I, et al. Association between microsomal triglyceride transfer protein gene polymorphism and the biological features of liver steatosis in patients with type II diabetes. Diabetologia. 2000;43:995-999.

42. Hsiao PJ, Lee MY, Wang YT, et al. MTTP-297H polymorphism reduced serum cholesterol but increased risk of non-alcoholic fatty liver disease-a cross-sectional study. BMC Med Genet. 2015;16:93.

43. Raabe M, Veniant MM, Sullivan MA, et al. Analysis of the role of microsomal triglyceride transfer protein in the liver of tissue-specific knockout mice. J Clin Invest. 1999;103:1287-1298.

44. Chang BH, Liao W, Li L, Nakamuta M, Mack D, Chan L. Liver-specific inactivation of the abetalipoproteinemia gene completely abrogates very low density lipoprotein/low density lipoprotein production in a viable conditional knockout mouse. J Biol Chem. 1999;274:6051-6055.

45. Xie Y, Newberry EP, Young SG, et al. Compensatory increase in hepatic lipogenesis in mice with conditional intestine-specific Mttp deficiency. J Biol Chem. 2006;281:4075-4086.

46. Gao S, He L, Ding Y, Liu G. Mechanisms underlying different responses of plasma triglyceride to high-fat diets in hamsters and mice: roles of hepatic MTP and triglyceride secretion. Biochem Biophys Res Commun. 2010;398:619-626.

47. Cuchel M, Meagher EA, du Toit Theron H, et al. Efficacy and safety of a microsomal triglyceride transfer protein inhibitor in patients with homozygous familial hypercholesterolaemia: a single-arm, open-label, phase 3 study. Lancet. 2013;381:40-46.

48. Raper A, Kolansky DM, Sachais BS, Meagher EA, Baer AL, Cuchel M. Long-term clinical results of microsomal triglyceride transfer protein inhibitor use in a patient with homozygous familial hypercholesterolemia. J Clin Lipidol. 2015;9:107-112.

49. Cuchel M, Rader DJ. Lipid-lowering treatment for homozygous familial hypercholesterolaemia-authors' reply. Lancet. 2013;381:1183.

50. Sacks FM, Stanesa M, Hegele RA. Severe hypertriglyceridemia with pancreatitis: thirteen years' treatment with lomitapide. JAMA Intern Med. 2014;174:443-447.

51. Burnett JR, Bell DA, Hooper AJ, Hegele RA. Clinical utility gene card for: abetalipoproteinaemia. Eur J Hum Genet. 2012;20. https://doi.org/10.1038/ejhg.2012.30.

52. Kawashiri MA, Tada H, Hashimoto M, et al. Extreme contrast of postprandial remnant-like particles formed in abetalipoproteinemia and homozygous familial hypobetalipoproteinemia. JIMD Rep. 2015;22: 85-94. 